\title{
Öffentliche Mobilität bewerten: Ansprüche an mobilitätsbezogene Mess- und Vergleichsverfahren
}

\section{Alexander Rammert}

\section{Einleitung}

Mobilität hat sich in den letzten Jahrzehnten vom ungeliebten Stiefkind zum ,Posterchild' einer modernen Verkehrspolitik und -planung gewandelt. Statt Verkehr zu planen wird jetzt Mobilität, gemanagt', die Verkehrsunternehmen werden zu Mobilitätsdienstleistern, es wird ein Grundrecht auf Mobilität gefordert und eine Vielzahl an Akteuren bietet jetzt sogar ,mobility as a service' an. Mobilität hat im öffentlichen Diskurs ebenso wie in planungspolitischen Debatten den Verkehr als zentrales Narrativ abgelöst. Moderne verkehrspolitische Leitbilder erheben individuelle Möglichkeiten und Lebensqualitäten zu neuen Zielgrößen; Verkehrsflussstärken und Infrastrukturausbaupläne verschwinden im Hintergrund: der Anspruch einer Öffentlichen Mobilität entsteht (siehe den Beitrag von Wolking). Mittlerweile hat die Mobilität in Deutschland sogar ihr erstes eigenes Gesetz (siehe den Beitrag von Kirchner in diesem Band) und läutet damit auch auf legislativer Ebene den Wandel ein. Jedoch - und dies ist kennzeichnend für jeden gesellschaftlichen Transformationsprozess - entsteht eine immer größer werdende Diskrepanz zwischen den Ansprüchen einer menschenorientierten Mobilitätsplanung und der tatsächlich praktizierten sowie institutionalisierten Verkehrsplanung (Schwedes 2018, S. 5). Sie beruht insbesondere in Deutschland noch immer auf den Normen der nachkriegszeitlichen Generalsverkehrsplanung, dominiert von damaligen Leitbildern wie der autogerechten Stadt oder dem Verkehr als Wirtschaftstreiber (Steierwald et al. 2005, S. 403 ff.). Dementsprechend befinden sich die aktuelle Politik und Planung in der unbefriedigenden Situation, dass die Mobilität zwar allseits als zentrale Planungsdimension

\author{
A. Rammert $(\square)$ \\ Technische Universität Berlin, Berlin, Deutschland \\ E-Mail: alexander.rammert@tu-berlin.de \\ (C) Der/die Autor(en) 2021 \\ O. Schwedes (Hrsg.), Öffentliche Mobilität, \\ https://doi.org/10.1007/978-3-658-32106-2_10
}


anerkannt wird, es jedoch an Institutionen und Instrumenten fehlt, diese evidenzbasiert zu gestalten. So ist es nicht möglich, mit den klassischen Verfahren der Wirkungsabschätzung und -berechnung die Mobilität adäquat abzubilden. Ja selbst die erlernten ingenieurswissenschaftlichen Kompetenzen der Verkehrsplanenden stoßen bei der Mobilität an ihre Grenzen, wenn Mensch und Umwelt statt Straße und Ampel zum Ansatzpunkt der Planung werden. Dies hatte zur Folge, dass in vielen Verwaltungen Deutschlands gänzlich neue Stellen für Mobilitätsmanager oder Mobilitätsbeauftragte durch das Forschungsministerium geschaffen wurden (BMBF 2019). Neue Kompetenzen sind gefragt, wie Mobilität zu untersuchen ist und wie mobilitätsbezogene Maßnahmen partizipativ umgesetzt werden können, denn andernfalls bleibt deren Wirkung auf die Mobilität zumeist eingeschränkt. Die Mobilitätsplanenden von morgen bauen nicht mehr lediglich Infrastrukturen oder programmieren Signalanlagen, sondern sie gestalten die Mobilität von Menschen und beeinflussen ihr Handeln. Doch nicht nur Ausbildung und Personal sind von diesem Wandel betroffen, auch die herkömmlichen Instrumente der Verkehrsplanung, wie Nutzen-Kosten-Analysen, Verkehrsflusszählungen oder Verkehrssystemmodellierungen, sind ungeeignet, um die Mobilität für Politik und Planung zielorientiert gestaltbar zu machen. Viel mehr braucht es neue Instrumente, die dezidiert die Mobilität messbar und planbar machen. Nur so kann die Mobilität ebenbürtig dem Verkehr und der Infrastruktur als Teil einer integrierten Politik und Planung institutionalisiert werden. Andernfalls bleibt die Mobilität als unkonkrete Gestaltungsgröße weiterhin eine Black Box für die Planungspraxis. Es benötigt also Instrumente, welche die Komplexität der Mobilität auflösen können, indem sie Transparenz über lokale Verhältnisse und normative Ansprüche herstellen. Was bedeutet eine , hohe Mobilität‘? Wann ist ein Mindestmaß an Mobilität gewährleistet und wie kann der Planende dies ermitteln? Die Mobilitätsforschung bietet hierfür mittlerweile ein breites Portfolio von zum Teil sehr aufwendigen Verfahren an, um die Mobilität der Menschen adäquat zu erfassen (Wilde et al. 2017) oder diese mit passenden Maßnahmen des Mobilitätsmanagements zu gestalten (Schwedes und Rammert 2020b). Was fehlt, ist ein großräumiges Bewertungsinstrument, das ana$\log$ zur Erreichbarkeitsanalyse oder Gesundheitsberichtserstattung die Mobilität von Kommunen und Städten messbar und vergleichbar macht. Wie sonst sollen Zielkriterien, wie eine hohe Mobilität oder ein Mindestmaß für Mobilität, systematisch überprüft werden? Die Herausforderung besteht also darin, die sozialen und damit qualitativen Ausprägungen von Mobilität quantitativ und aggregiert darstellbar zu machen (siehe auch den Beitrag von Mietzsch in diesem Band). Dafür bietet die klassische Verkehrs- und Mobilitätsforschung bis jetzt noch keine Lösung, weshalb ein Blick auf benachbarte Disziplinen hilfreich 
erscheint. So existiert beispielsweise in der vergleichenden Politikwissenschaft eine Methodik, die es ermöglicht, komplexe soziokulturelle Zusammenhänge simplifiziert und aggregiert auf globaler Ebene darzustellen: der Index. Er dient als sozialwissenschaftliches Instrument um komplexe Zusammenhänge sichtbar zu machen und in Folge dessen auch politische und gesellschaftliche Veränderungen anzustoßen. Die Indexkonstruktion könnte am Ende auch ermöglichen die Mobilität, ebenso wie Bildung, Demokratie oder Gerechtigkeit, transparent und damit bewertbar zu machen. Eine Eigenschaft, die der praktizierten Mobilitätsplanung bis heute fehlt.

Im Folgenden wird skizziert, wie der Weg zu einem wissenschaftlichen Mobilitätsindex aussehen muss: Angefangen vom Verständnis, wie Mobilität überhaupt als adäquate Planungsgröße aufgefasst werden kann, hin zu einer detaillierteren Betrachtung von Indizes als politisches und planerisches Bewertungsinstrument. Damit grenzt sich der hier vorgestellte Ansatz für eine Indizierung von Mobilität explizit von bereits existierenden, reduktionistischen ,Mobilitätsindizes' ab, deren wesentlicher Inhalt darin besteht, verkehrsträgerspezifischen Aspekte (Mobilitätsindex der Allianz pro Schiene) oder wirtschaftliche Rahmenbedingungen (Urban Mobility Index) abzubilden, mit jeweils nur ein Bruchteil des komplexen wissenschaftlichen Mobilitätsverständnisses erfasst wird. Arrondiert werden diese Erkenntnisse in den Ansprüchen an die wissenschaftliche Mobilitätsmessung. Hier wird detailliert dargestellt, welche Faktoren es in solch einer Messung einzubeziehen gilt, welche Gütekriterien gelten und mit welchen Einschränkungen umgegangen werden muss. Ein Mobilitätsindex, so die These dieses Textes, kann im Ergebnis sowohl für den planungspolitischen Anspruch einer Öffentlichen Mobilität als auch für die Selbstermächtigung der Zivilgesellschaft genutzt werden. Damit bietet der Mobilitätsindex Planung und Politik ein längst überfälliges Werkzeug, die Mobilität bewertbar zu machen und vergleichbar darzustellen.

\section{$1 \quad$ Mobilität als Planungsgröße}

Zunächst wollen wir uns die Mobilität als sozialräumliches Phänomen näher anschauen, um zu klären, in welcher Form sie überhaupt als Planungsgröße genutzt werden kann. So existiert der Mobilitätsbegriff zwar seit über 100 Jahren, in der ingenieurswissenschaftlichen Planungspraxis spielte er jedoch lange Zeit für die Planenden eine ähnlich nachgeordnete Rolle wie ,Wohlbefinden " oder, Ästhetik'. Dieser Zusammenhang lässt sich über die Geschichte der Verkehrsplanung in Deutschland auch nachvollziehbar nachzeichnen. So bestand 
zunächst die Aufgabe der nachkriegszeitlichen (Straßen-)Verkehrsplanung maßgeblich darin, Infrastrukturen für die wachsenden Verkehrsströme ausreichend zu dimensionieren (Mäcke 1964). Der Mensch wurde unter den Verkehrsmitteln subsummiert und so transformierten die Planenden menschliche Bewegungsmuster zu objekthaften Verkehrsflüssen, analog der Fließbandlogistik in den wachsenden Industrieanlagen. Entsprechend spielten bei der Infrastrukturplanung verkehrstechnische Größen wie Geschwindigkeit, Durchflusskapazität und Materialabnutzung eine maßgebende Rolle; der Mensch galt allenfalls als Störfaktor, der den gleichgeschalteten Verkehrsfluss durch seine Irrationalität behinderte (Knoflacher 2009, S. 71). ${ }^{1}$ Mit zunehmendem Verkehrswachstum und steigender Verkehrsbelastung für Städte in den 1970er und 1980er Jahren brauchte die Verkehrsplanung neue Instrumente, um gestalterisch in das System eingreifen zu können. Mit der zunehmenden Computerisierung ergaben sich für die damaligen Ingenieure neue technische Möglichkeiten das Verkehrssystem zu beeinflussen, ohne großflächige, bauliche Maßnahmen ergreifen zu müssen. Neben Bauingenieuren gesellten sich so mit der Zeit immer mehr Informationstechniker zur Verkehrsplanung und entwarfen mit dem Verkehrsmanagement eine völlig neue Planungsdimension für den Verkehr (Vallée 2008). Neuartige Regelungsschaltungen und komplexe Verkehrsleitsysteme dominierten schon bald sämtliche Verkehrsanlagen und versuchten die weiterwachsenden Verkehrsströme möglichst effizient zu organisieren. Mittlerweile wurde die Irrationalität des Menschen als gegeben hingenommen und in Form von mathematischen Konstanten, wie ,Befolgungsraten“ oder ,Zufallsaktoren', in verkehrstechnischen Formeln und Modellen operationalisiert. Grüne Wellen, dynamische Seitenstreifenfreigabe oder systematische Verkehrsfunkmeldungen eröffneten der Verkehrsplanung neue Maßnahmenfelder, den Verkehr und seine Effekte in neuartiger Weise gestalten zu können: vergeblich. Heute - 70 Jahre nach Beginn der Generalsverkehrsplanung und vielzähligen Innovationen in baulichen, organisatorischen und informationstechnischen Maßnahmen - befinden wir uns weiterhin am temporären Maximalpunkt des Verkehrsaufkommens und den damit einhergehenden negativen Effekten (Schwedes und Rammert 2020b, S. 5-13). Infrastruktur- und Verkehrsmanagement vermochten es nicht, dem ungebändigten Wachstum des Verkehrs auf Kosten von Mensch und Natur Einhalt zu gebieten.

\footnotetext{
${ }^{1}$ Interessanterweise taucht dieses historische Verständnis von Mensch und Verkehr auch wieder im Zusammenhang mit autonomen Verkehrssystemen auf. Aus technischer Sicht ist auch hier der Einfluss des Menschen möglichst zu minimieren, um eine höhere Transporteffizienz zu erreichen (Schlag 2016).
} 
Einzig das wirtschaftliche Wachstum wies kontinuierlich eine positive Bilanz auf und gilt damit bis heute als wesentliches Begründungsargument der klassischen Verkehrsplanung. Doch die Narrativen haben sich geändert: Mensch und Umwelt rücken in das Zentrum internationaler Leitbilder (FES 2019, S. 5 ff.) und auch die Verkehrsplanung kann mit wirtschaftlichen Mehrwerten nicht mehr die negativen Auswirkungen auf Mensch, Natur und Klima kaschieren. Und so rückte mit der Zeit die Mobilität zunehmend in den Blick der Verkehrsplanung und mit ihr die Sozialwissenschaften.

Betrachten wir diese Entwicklung der deutschen Verkehrsplanung in der Retrospektive, wird schnell offensichtlich, weshalb die Mobilität als neuste Dimension einer integrierten Verkehrsplanung (Schwedes et al. 2018) es bis heute so schwer hat, als vollwertige Planungsgröße in der Praxis zu fungieren. Die jahrzehntelange Ausrichtung von Verfahren, Prozessen und Strukturen an den ingenieurs- und technikwissenschaftlichen Anforderungen der Verkehrssysteme hat dazu geführt, dass ein vollständiges Unverständnis der klassischen Verkehrsplanung gegenüber mobilitätsbezogenen Belangen herrscht. Belege hierfür sind unter anderem die Lehrcurricula der größten deutschen Verkehrsplanungsstudiengänge in Dresden, Berlin, Braunschweig und Aachen sowie die schwerfällige Integration von Mobilitätsmanagement in der Verwaltungspraxis (Schwedes et al. 2017, S. 61 ff.). Auch die Regelwerke und die Konstitution der deutschen Forschungsgesellschaft für Straßen- und Verkehrswesen (FGSV) bringt dieses Defizit zum Ausdruck (siehe Abb. 1).

Dies hat zur Folge, dass die Mobilität lange Zeit anderen Disziplinen wie der Geographie, der Stadtplanung oder der Soziologie überlassen wurden. Dies ist insofern problematisch, da weder die sozialwissenschaftlich geprägten noch die ingenieurswissenschaftlich geprägten Disziplinen sich um eine adäquate Operationalisierung der Mobilität und Mobilitätsplanung bemühten (Wilde und Klinger 2017). Daraus folgt die heute paradoxe Situation, dass Mobilität auf der einen Seite als zentrale Planungsgröße beschworen wird und quer durch die Republik neue Verwaltungsstellen zu dessen Gestaltung entstehen, auf der anderen Seite es aber weder ein klares Verständnis davon gibt, was Mobilität überhaupt ist, noch adäquate Regelwerke und Planungsleitfäden bestehen, wie sie zu gestalten wäre (Schwedes und Rammert 2020b). An dieser Stelle ist darauf hinzuweisen, dass dieses Paradox ein spezifisches Phänomen für Deutschland ist. In fast allen europäischen Nachbarländern existieren entweder Adaptionen der europäischen Sustainable Urban Mobility Plans (Kiba-Janiak und Witkowski 2019) oder eigene Planungsinstrumente, wie die Mobilitätspläne in den Niederlanden oder Frankreich (Wulfhorst und Wolfram 2010). Weshalb insbesondere Deutschland so resistent gegenüber einer umfassenden Integration von Mobilität 


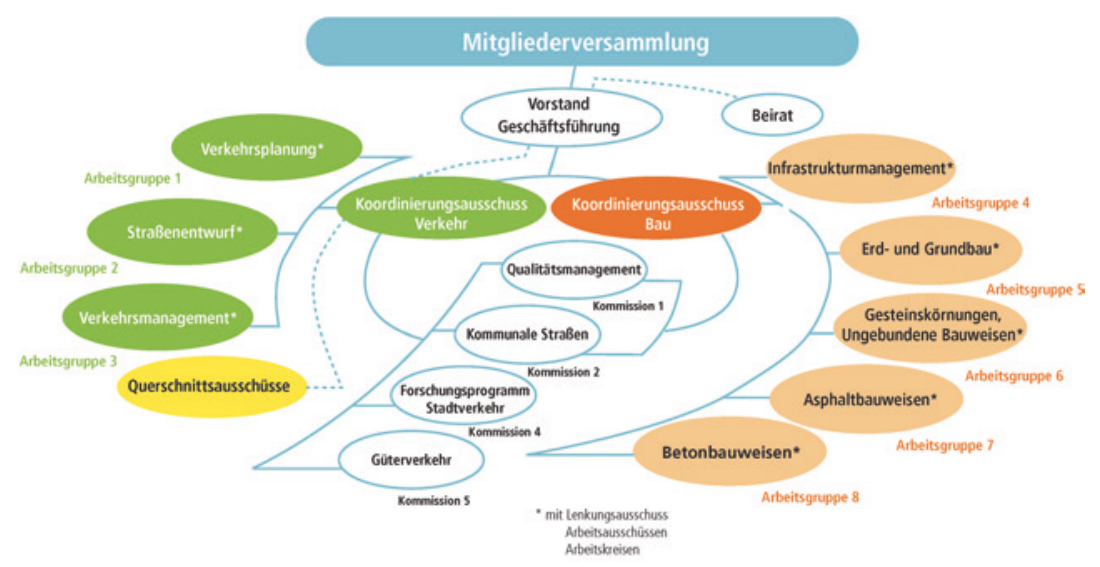

Organigramm der FGSV

Abb. 1 Kennzeichnend für die monothematische Ausrichtung ist das Organigramm der Forschungsgesellschaft für Straßen- und Verkehrswesen, welche de facto alle relevanten deutschen Regelwerke zur Planungsintegration von Mobilität und Verkehr erstellt (FGSV 2020)

in der Planung ist, muss zukünftig noch untersucht werden. Fest steht jedoch, dass die hier dominierenden Verkehrsentwicklungspläne sowie die GatekeeperFunktion der FGSV in Bezug auf Regelwerke und Planungsinstrumente wesentliche Faktoren diesbezüglich darstellen (Schwedes und Rammert 2020a). Die aktuell entstehenden Mobilitätspläne ${ }^{2}$ und Mobilitätsgesetze, sowie die Hinweise zu Mobilität und sozialer Exklusion der FGSV (2015), sind erste Lichtblicke für den ansonsten im Dunkeln tappenden deutschen Mobilitätsplanenden. So bleibt festzuhalten, dass aus historischen, strukturellen und kulturellen Gründen die Mobilität in Deutschland als Planungsgröße weiter marginalisiert bleibt. Gleichzeitig steigt der Bedarf vonseiten der Planung und Politik, Mobilität evidenzbasiert gestalten zu können. Es braucht also neben einem klaren Verständnis, was Mobilität eigentlich ist, Verfahren und Instrumente diese messen und bewerten zu können. ${ }^{2}$ Positive Beispiele für Mobilitätspläne finden sich unter anderem für die Städte München,
Kassel, Osnabrück, Düsseldorf und die Region Frankfurt. 
Bevor wir uns im Folgenden die Indizes als planungspolitisches Instrument näher anschauen, gilt es die Mobilität als Planungsgröße klar abzugrenzen. Andernfalls ist es für jegliches Planungsverfahren kaum möglich, zielorientiert und evidenzbasiert ein Phänomen wie die Mobilität zu gestalten.

Zunächst lässt sich festhalten, dass selbst in der Wissenschaft bis heute keine Einigkeit darüber herrscht, was genau die Mobilität beschreibt. Vielmehr verfügen die verschiedenen Wissenschaftsdisziplinen jeweils über ihr eigenes Mobilitätsverständnis, wodurch eine integrierte Zusammenführung ingenieurs-, planungs- und sozialwissenschaftlicher Aspekte erschwert wird. Solch eine Zusammenführung erscheint jedoch sinnvoll, da Mobilität - und hierin sind sich die unterschiedlichen Disziplinen einig - von vielen unterschiedlichen Faktoren beeinflusst werden kann. Dazu gehören räumliche und verkehrliche Strukturen, die klassisch in den Raum- und Verkehrswissenschaften untersucht werden, soziale und kulturelle Strukturen, welche in der Soziologie eine Rolle spielen, soziodemografische Faktoren, die häufig in den Geographischen Wissenschaften beforscht werden, oder subjektive Wahrnehmungen, die in der Psychologie und Verhaltensforschung von zentraler Bedeutung sind. Wir sehen: Mobilität ist ein interdisziplinäres Phänomen und kann deshalb auch nur disziplinübergreifend für die Planungspraxis operationalisiert werden.

Ausgehend vom Verständnis, dass Mobilität die subjektiven Möglichkeiten für Ortsveränderungen und Bedürfniserfüllung beschreibt (Schwedes et al. 2018, S. 5), können wir in den unterschiedlichen Disziplinen mit der Suche nach möglichen Einflussfaktoren für diesen individuellen Möglichkeitsraum beginnen. Ziel ist es, alle mobilitätsrelevanten Indikatoren zu identifizieren, um diese später in ihrer Gesamtheit für mögliche Planungsinstrumente nutzbar zu machen. Hierfür bieten Raum- und Verkehrswissenschaften mit der Erreichbarkeitsanalyse bereits ein funktionierendes Planungsinstrument, um räumliche Strukturen in Bezug auf die Erreichbarkeit von Grundversorgungseinrichtungen, wie Lebensmittelgeschäften, Arztpraxen oder Bildungseinrichtungen, messbar zu machen (Schwarze 2015). Bezogen auf die Mobilität, als subjektive Möglichkeit für Ortsveränderung und Bedürfniserfüllung, ist somit eindeutig, dass die räumlichen Rahmenbedingungen, welche in den Erreichbarkeitsanalysen verwendet werden, auch für die Untersuchung von Mobilität eine Rolle spielen müssen. Aber die Erreichbarkeit hat nicht nur eine räumliche, sondern auch eine individuelle Komponente (Altenburg et al. 2009). So ist beispielsweise die fußläufige Erreichbarkeit von einem Lebensmittelgeschäft nur gegeben, wenn das Individuum zu Fuß gehen kann. Ebenso wie die MIV-Erreichbarkeit (BBSR 2020) nur für diejenigen gilt, die sich einen privaten Pkw leisten können. Insofern gibt es auch eine individuelle Komponente, die darüber entscheidet, ob die räumlichen 
und verkehrlichen Strukturen überhaupt genutzt werden können. Für diese sogenannten individuellen Handlungsvoraussetzungen existieren in Deutschland bereits Messinstrumente. In den großen deutschen Querschnittstudien Mobilität in Deutschland - MiD (Nobis und Kuhnimhof 2017) und Mobilität in Städten - SrV (Ahrens et al. 2014) werden viele dieser soziodemografischen Faktoren, wie Haushaltseinkommen, Verkehrsmittelzugänge oder gesundheitliche Einschränkungen, erfasst. Jedoch greift beispielsweise die Messung von Mobilität der MiD zu kurz, wenn die Mobilitätsquote einzig über den „Anteil der Personen, die an einem Tag mindestens einmal aus dem Haus gehen“ (Nobis und Kuhnimhof 2017, S. 25) definiert wird. Diese Reduktion der Mobilität auf tatsächlich durchgeführte Ortsveränderungen, also Verkehr, wird in der anschließenden Analyse der Daten weiter verstärkt, indem zwischen „mobilen“ und ,nicht mobilen“ Menschen unterschieden wird, die sich einzig dadurch unterscheiden, ob diese am Stichtag aus dem Haus gegangen sind (ebd., S. 25 ff., 131). Es zeigt anschaulich, wie rudimentär das Mobilitätsverständnis bei einer der größten deutschen Querschnittstudien ist, da die gemessene ,Außerhäusigkeit ‘ nur einen winzigen Bruchteil der tatsächlichen Mobilität der Menschen darstellt. Trotzdem erfasst die Studie viele wichtige soziodemografische Faktoren, die es für eine adäquate Messung von Mobilität zu beachten gilt. Sie verpasst es aber leider, diese mit den individuellen Möglichkeitsräumen zu kontextualisieren. Doch nicht nur die Erreichbarkeit, sondern auch die Umweltstrukturen sind relevante Faktoren, die in den Verkehrswissenschaften zwar wenig Beachtung finden, in anderen Disziplinen aber maßgeblich die Möglichkeitsräume der Menschen beeinflussen. Eine hohe Luftbelastung, ein erhöhter Lärmpegel oder eine starke thermische Belastung können ebenso auf die individuellen Möglichkeiten einwirken wie die Entfernung zur Bushaltestelle (Becker 2018, S. 75). Hierzu haben die Umweltwissenschaften mit der Umweltgerechtigkeitsanalyse ebenfalls ein Instrument entwickelt, das bereits erfolgreich in der Berliner Verwaltung integriert werden konnte (SenStadtUm 2017). Hier zeigt sich mitunter, wie zwar viele mobilitätsrelevante Faktoren von unterschiedlichen Fachdisziplinen gemessen und in der Fachplanung verwendet werden, die Anwendung jedoch immer dispers und segregiert stattfindet. Eine Mobilitätsmanagerin müsste demnach, unter anderem, all diese Verfahren beherrschen und anwenden können; ein entsprechend integriertes Ausbildungsprogramm existiert bis heute an den deutschen Universitäten aber noch kaum. ${ }^{3}$

${ }^{3}$ Eine Ausnahme stellt der Studiengang „Mobilitätsmanagement“ an der Hochschule RheinMain dar. 
Doch neben den räumlichen und soziodemografischen Faktoren konnten andere Disziplinen noch weitergehende Einflüsse identifizieren, welche die individuellen Möglichkeiten zur Ortsveränderung und Bedürfniserfüllung beeinflussen. So entdeckten die Raumwissenschaften schon sehr früh, dass die Mobilität und die daraus resultierenden Entscheidungen für Wohnstandort und Arbeitsplatz stark mit den sozialökonomischen Strukturen zusammenhängen (Jarass 2012; Scheiner 2016). Dies bedeutet, dass beispielsweise ein attraktiver Arbeitsplatz im Nachbarort oder die Vergünstigung des Arbeitswegs durch die Entfernungspauschale selbstverständlich die individuelle Mobilität verändern können (Holz-Rau 2019). Weiterhin zeigt uns die Armutsforschung, dass sozialstrukturelle Elemente, wie eine Mindestsicherung oder Sozialtickets, wichtige Komponenten sind, um die Möglichkeitsräume der betroffenen Menschen nicht unter ein Mindestniveau fallen zu lassen (Daubitz 2017). Auch hierfür hat die Armutsforschung mit der Lebenslagenuntersuchung bereits ein Instrument entwickelt, wie diese Indikatoren auf Bundesebene in der Planung operationalisiert werden können (Voges et al. 2003). Neben diesen sozialstrukturellen Einflüssen konnten aber auch auf individueller Ebene Faktoren identifiziert werden, welche die Mobilität nachhaltig beeinflussen. Insbesondere die Humangeographie hat hier durch ihre kultur- und sozialwissenschaftlichen Perspektiven wichtige Zusammenhänge aufgezeigt. So lassen sich mit den Lebensstilen und den sozialen Milieus zwei wesentliche Untersuchungsgrößen identifizieren, die Einfluss auf die individuellen Möglichkeitsräume nehmen (Beckmann et al. 2006). Bezüglich dieser soziokulturellen Handlungsvoraussetzungen mangelt es noch am ehesten an funktionierenden Operationalisierungen für die Planungspraxis, auch wenn mit den Mobilitätsstilen (Götz et al. 2016) und Untersuchungen zu Mobilitätsmilieus (Dangschat 2018) erste Ansätze existieren, diese Erkenntnisse für eine Mobilitätsplanung nutzbar zu machen. Zusammenfassend zeigt sich ein weites Feld an unterschiedlichen Perspektiven auf die Mobilität und den damit verbundenen Fachdisziplinen. Einige der Erkenntnisse konnten bereits isoliert in disziplinspezifischen Planungsinstrumenten operationalisiert werden, ein integriertes Planungsmodell für Mobilität fehlt jedoch weiterhin. Insbesondere fehlt all diesen Ansätzen ein zentrales Element, was die Mobilität grundlegend konstituiert: die subjektive Wahrnehmung.

Ausgehend von der Annahme, dass die Mobilität die subjektiven Möglichkeiten für Ortsveränderung und Bedürfniserfüllung beschreibt, müssen selbstverständlich bei einer adäquaten Operationalisierung auch die subjektiven Wahrnehmungen und Empfindungen der Menschen berücksichtigt werden. So ist die Subjektivität das zentrale Unterscheidungsmerkmal, welches Mobilität von Verkehr, Erreichbarkeit oder der Lebenslage unterscheidet (Busch-Geertsema et al. 2016, S. 762). Zumindest in diesem Punkt sind sich die meisten Sozialund Planungswissenschaften einig, auch wenn die Auffassung divergiert, ob und 
wie diese subjektiven Komponenten von Mobilität in der Praxis operationalisiert werden können (Kutter 2010; Wilde und Klinger 2017, S. 17). Auch wird deutlich, dass die subjektiven Komponenten von Mobilität weitaus schwieriger auf diskrete Indikatoren aufzuteilen sind als beispielsweise soziodemografische Faktoren, wie Alter, Einkommen und Berufsstand. Dieser Zusammenhang lässt sich als einer der Hauptgründe anführen, weshalb Mobilität bis heute in der Planungspraxis so schwerfällig gehandhabt wird, bzw. breite Untersuchungen der Mobilität, wie die MiD, reduktionistisch nur räumliche und individuelle Faktoren mit einbeziehen: sie lassen sich einfach besser quantifizieren. Auch deshalb wurde im Zuge einer Ausweitung von Mobilitätsmanagement immer wieder gefordert, eine quantitative Betrachtung der tatsächlichen Mobilität zu ermöglichen (Schwedes et al. 2017, S. 165 ff.).

Insbesondere im deutschsprachigen Raum lassen sich hierzu kaum Beispiele identifizieren, sehr wohl aber im internationalen Bereich. So wurden beispielsweise subjektive Mobilitätsfaktoren in Form der unterdrückten Nachfrage von Mobilität (Hine und Grieco 2003), der Untersuchung zur Zufriedenheit mit der individuellen Erreichbarkeit (Currie et al. 2010) sowie einer Untersuchung der Zufriedenheit mit der eigenen Mobilität (Spinney et al. 2009) operationalisiert. Hierbei wird in der Regel von der ,subjektiven Erreichbarkeit“" (Scheepers et al. 2016, S. 96) oder den „selbstberichteten Schwierigkeiten bei Ortsveränderungen“ (Delbosc und Currie 2011, S. 172) gesprochen. Weiterhin gibt es bereits mit der „Perceived Accessibility Scale“ in Schweden (Lättman et al. 2016, S. 257) oder den „Transport Disadvantage“-Untersuchungen in Australien (Currie et al. 2010) Versuche, diese beiden Begrifflichkeiten in der Praxis der Mobilitätsplanung zu operationalisieren und damit messbar zu machen. In den Untersuchungen konnten verschiedene Frage-Items identifiziert werden, welche valide Aussagekraft bezüglich der wahrgenommenen Erreichbarkeit und des daraus resultierenden Möglichkeitsraums haben (Delbosc und Currie 2011, S. 172; Lättman et al. 2016, S. 261). Aber auch weitere Faktoren, wie die individuelle Lebenszufriedenheit oder die Wahrnehmung des familiären und sozialen Umfelds, spielen für die persönliche Mobilität eine wichtige Rolle (Nordbakke und Schwanen 2015). Auch hierzu gibt es im Rahmen der Exklusionsforschung verschiedene Beispiele, wie diese Phänomene durch Indikatoren operationalisiert werden können. $\mathrm{Zu}$ nennen sind Instrumente wie der „Personal Wellbeing Index“ (DeVos et al. 2013, S. 425) oder der „Social Exclusion Index“ (Stanley et al. 2011, S. 796), welche die subjektive Wahrnehmung von soziokulturellen Faktoren über Befragungsitems für die praktische Forschung operationalisieren. Damit zeigen insbesondere die internationalen Sozialwissenschaften auf, welche Möglichkeiten existieren, auch 
subjektive Wahrnehmungen von Raum und Gesellschaft für die Planung messbar zu machen.

Zusammenfassend zeigt sich, dass die Mobilität in ihrer Komplexität durch vielfältige Einflussgrößen bestimmt wird. Passend dazu haben sich eine Vielzahl an wissenschaftlichen Disziplinen mit der Erforschung und Messung einzelner Einflussdimensionen beschäftigt. In Hinblick auf eine Integration der Mobilität als Planungsgröße für die Praxis erscheint es demnach naheliegend, all diese verschiedenen Einflussdimensionen in einem systemischen Modell für Mobilität zusammenzuführen. Dieses Mobilitätsmodell stellt die Grundlage dar, um im Anschluss unterschiedliche Mess-, Bewertungs- oder Beeinflussungsinstrumente für die Planungspraxis zu entwerfen. Nach einer interdisziplinären Meta-Analyse deutscher und internationaler Mobilitätsforschungen ${ }^{4}$ lässt sich zunächst festhalten, dass zwei Ebenen zu unterscheiden sind: Erstens die objektiven Möglichkeiten für Individuen, sich im Raum zu bewegen und Bedürfnissen nachzugehen. Zweitens die subjektiv wahrgenommenen Möglichkeiten, welche die objektive Ebene mit den individuellen Wahrnehmungen und Erfahrungen kontextualisiert. Anschaulich wird das Zusammenspiel dieser beiden Ebenen anhand von verkehrsplanerischen Beispielen wie Bushaltestellen oder Fußgängerunterführungen. Die objektive Verfügbarkeit dieser Infrastrukturen gibt nur teilweise Aufschluss über die tatsächliche Erreichbarkeit. Menschen, denen das Wissen um die Haltestellen fehlt, deren Ziele von dem Bus nicht angesteuert werden, oder Ängste sie vom Nutzen der Fußgängerunterführungen abhalten, haben subjektiv eine viel geringere Erreichbarkeit, als auf objektiver Ebene suggeriert wird. Ihre Mobilität ist also eingeschränkt und kann in diesem Fall nicht ausschließlich durch verkehrsstrukturelle Maßnahmen erhöht werden.

Weiterhin muss auf objektiver Ebene zwischen strukturellen Rahmenbedingungen und individuellen Handlungsvoraussetzungen unterschieden werden. Dies bedeutet, dass bereits die objektive Ebene individuell kontextualisiert werden muss, um den tatsächlichen objektiven Möglichkeitsraum von Menschen zu bestimmen. Deutlich wird dies beispielsweise anhand der Barrierefreiheit. So ist es offensichtlich, dass ein junger Student und eine geheingeschränkte Seniorin aufgrund ihrer individuellen Voraussetzungen bereits aus objektiver Sicht komplett unterschiedliche Möglichkeitsräume besitzen. Die subjektiven Wahrnehmungen der Personen können diese Unterschiede noch weiter verstärken, aber

\footnotetext{
${ }^{4}$ Die besagte Meta-Analyse wurde im Rahmen des Promotionsvorhabens zum Mobilitätsindex durchgeführt und ist zum jetzigen Zeitpunkt noch nicht veröffentlicht.
} 
auch reduzieren. Die individuelle Mobilität setzt sich also im Ganzen aus drei zentralen Einflussdimensionen zusammen:

1. Die strukturellen Rahmenbedingungen

2. Die individuellen Handlungsvoraussetzungen

3. Die subjektiven Wahrnehmungen

Zusätzlich gilt für alle drei Teildimensionen der Mobilität, dass diese sowohl von räumlichen Faktoren als auch von soziokulturellen Faktoren beeinflusst werden. Es reicht also nicht die alleinige Betrachtung der Verkehrsverbindung zum Arbeitsplatz, des verfügbaren Haushaltseinkommens oder der wahrgenommenen Erreichbarkeit, sondern auch die bestehenden Subventionsstrukturen, die individuellen Lebensstile sowie die Wahrnehmung kultureller Normen müssen für eine vollwertige Betrachtung der Mobilität einbezogen werden. Erst dann kann die Planung den Anspruch erheben, tatsächlich Mobilitätsmanagement, Mobilitätsplanung oder Mobilitätsberichterstattung zu betreiben. Leider ist dies, bis auf wenige Ausnahmen in Deutschland (siehe den Beitrag von Hausigke und Kruse in diesem Band), bisher nicht gelungen, obwohl mit der ,nachhaltigen urbanen Mobilitätsplanung“ (SUMP) ein starkes europäisches Leitnarrativ existiert, dies alsbald zu ermöglichen (Rupprecht Consult 2020).

Für eine erfolgreiche Operationalisierung der Mobilität in Politik und Planung müssen somit alle drei Einflussdimensionen mit einbezogen werden (siehe Abb. 2). Erst im Anschluss sind evidenzbasierte Aussagen zur Mobilität, den daraus resultierenden Routinen und dem zu beobachtenden Mobilitätsverhalten

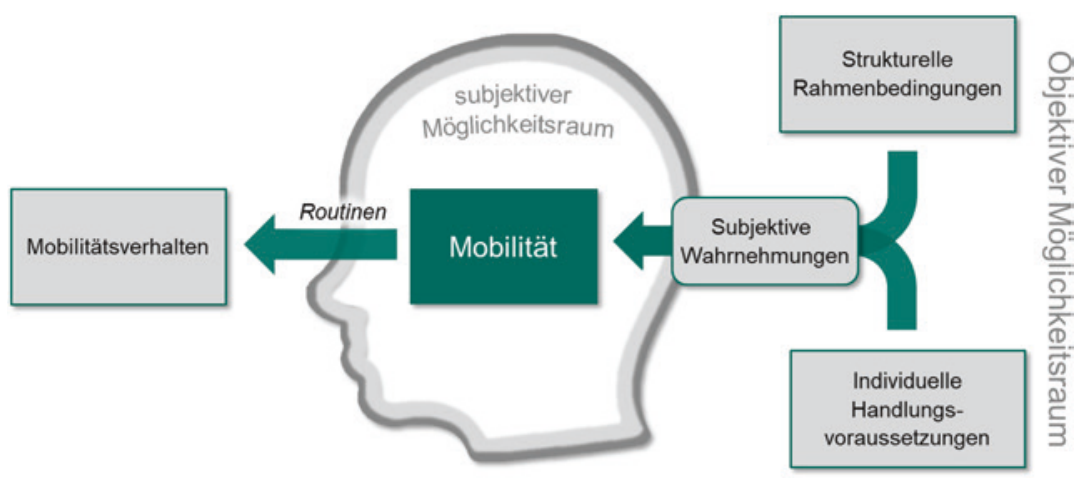

Abb. 2 Modell der Einflussdimensionen von Mobilität. (Eigene Darstellung) 
möglich. Dies ist bis dato innerhalb der existierenden Mobilitätsmessungen weder bei den Querschnittsumfragen noch bei den Erreichbarkeitsuntersuchungen in Deutschland der Fall. Wir benötigen also ein praxistaugliches Instrument, das es ermöglicht, eine Vielzahl dieser zum Teil sehr unterschiedlichen Indikatoren in einem Verfahren zusammenzuführen.

\section{Indizes als politisches Bewertungsinstrument}

Eine Methode, die es erlaubt komplexe soziokulturelle Phänomene, wie beispielsweise die menschliche Entwicklung, Meinungsfreiheit oder Demokratie, $\mathrm{zu}$ messen und normativ $\mathrm{zu}$ bewerten ist die Indexbildung. Ursprünglich eher dem politik- und wirtschaftswissenschaftlichen Bereich zuzuordnen, kommt der Index als sozialwissenschaftliche Methodik immer häufiger bei der Darstellung struktureller und kultureller Faktoren zur Anwendung (Pickel und Pickel 2012, S. 7). Zu seinen bekanntesten Vertretern gehört der Human Development Index, der von den Vereinten Nationen weltweit erhoben und ausgewertet wird (UN 2018). Aber auch andere Indexformen, wie der Bertelsmann Transformation Index, der Corruption Perception Index oder der Better Life Index der OECD, werden international genutzt, um komplexe Phänomene quantifizieren zu können und eine Planungsgrundlage $\mathrm{zu}$ deren Beeinflussung $\mathrm{zu}$ schaffen. Dies macht den Index zu einer geeigneten Methode für die Bewertung von Mobilität, da es sich ebenfalls, wie bereits zuvor dargestellt, um ein soziales, strukturelles und kulturelles Phänomen handelt. Der Begriff des Index beschreibt „,die Zusammenfassung mehrerer Einzelindikatoren zu einer neuen Variablen nach festgelegten Vorschriften“ (Latcheva und Davidov 2014, S. 745). Diese ,neue Variable“ zeichnet sich besonders dadurch aus, dass sie selbst nicht direkt beobachtbar und damit nicht messbar ist - ein wesentliches Merkmal von Mobilität. Es erscheint also zielführend auch die Mobilität mithilfe eines Index zu operationalisieren, um damit Politik und Planung die Möglichkeit zu geben, Mobilität großräumig messbar zu machen.

Die Indexkonstruktion ist zunächst ein Auswertungsverfahren und kein eigenständiges Erhebungs- oder Messverfahren. Nichtsdestotrotz spielen die Erhebung und Validierung von Daten im Rahmen der Indikatorauswahl eine tragende Rolle, die maßgeblich über Aussagekraft und Qualität des anschließenden Index entscheiden. Ein Index ist in diesem Sinne eine Maßzahl für das relative Verhältnis mehrerer Indikatoren im Zeitverlauf oder im gegenseitigen Vergleich (Atteslander 2010, S. 227). So besitzt ein einzelner Index ohne Kontext nur begrenzte Aussagekraft, entfaltet er doch erst im Vergleich seine wahre Funktion 
als Bewertungsinstrument. Hierbei ist zu beachten, dass Bewertungsinstrumente immer eine normative Komponente besitzen. So ist innerhalb der jeweiligen Indexmethodik chiffriert, was eine gute menschliche Entwicklung, eine gute Demokratie oder eben eine hohe Mobilität ausmacht. Dadurch bietet sich der Index durch seine Konstitution vor allem als politisches Benchmarkingtool für die Performance von Regionen und Ländern an (OECD 2008, S. 13); besitzt also immer einen räumlichen oder zeitlichen Bezug. Indizes ermöglichen uns Erkenntnisse über Syndrome, also der multikausalen Kollektion von Phänomenen, gesellschaftlicher und politischer Zustände (Pickel und Pickel 2012, S. 2), welche durch einzelne Indikatoren oder Messwerte nicht abgebildet werden können. Zentrales Element eines Index ist die Aggregation verschiedener Indikatoren zu einer Größe nach festgelegten Regeln. Diese sogenannten Aggregationsregeln definieren den quantitativen Prozess mit Rechenoperationen und Gewichtungen, welcher die Einzelindikatoren zu einer Zielvariablen zusammenführt.

Der Vorteil von Indizes gegenüber reinen Gruppierungen von Indikatoren sind neben der besseren Übersichtlichkeit auch die Erhöhung der Messgenauigkeit durch die Kombination mehrerer Variablen zur Messung eines theoretischen Begriffs (Latcheva und Davidov 2014, S. 748). Dies wird dadurch erreicht, dass verschiedene Messungenauigkeiten bei den einzelnen Indikatoren gegeneinander aufgewogen werden, wodurch im Indexkonstrukt eine Reduktion der Messfehler stattfindet (Kromrey et al. 2016, S. 171 ff.). Bereits der Einbezug von zwei verschiedenen Indikatoren reduziert den zufälligen Messfehler, der Einbezug von drei Indikatoren reduziert sogar systematische Messfehler (Latcheva und Davidov 2014, S. 749). Ein weiterer Vorteil des Index, der sich durch eine Verdichtung von Informationen und einer damit einhergehende Komplexitätsreduktion ergibt, ist die Vermeidung von Multikollinearität (Pickel und Pickel 2012, S. 1). Multikollinearität bedeutet, dass zwei oder mehr Indikatoren eine starke Korrelation miteinander aufweisen. Beispielweise ist zwischen den Indikatoren ,durchschnittliche Lebenserwartung ' und ,Ausprägung des Gesundheitssystems ' von einer starken Korrelation auszugehen, weshalb sie in einer reinen, nicht aggregierten Indikatorenliste nicht auf gleicher Ebene für beispielsweise den menschlichen Entwicklungsstand stehen sollten. Der Index bietet hierbei die Möglichkeit korrelierende Indikatoren entsprechend zu gewichten oder ganz zu exkludieren und damit eine Multikollinearität vollständig auszuschließen. Dies zeigt eindrücklich das Beispiel des Human Development Index, der am Ende die gesamte Facette der menschlichen Entwicklung auf nur vier Kernindikatoren reduziert (UN 2018).

Neben den genannten Vorteilen ist eine Indexkonstruktion aber auch mit Risiken beziehungsweise Nachteilen verbunden, welche mit dem quantitativen Simplifizierungsprozess einhergehen (OECD 2008, S. 13 f.). Das erste Problem 
von Indizes besteht darin, dass der Nutzende sich in der Regel nicht über die zugrundeliegenden Methoden und Informationen bewusst ist. Sie werden im Aggregationsprozess zwangsläufig verkürzt und bieten somit Platz für Interpretationsspielräume. So wissen beispielsweise die meisten Leserinnen und Leser über die Aussagelogik eines Human Development Index oder eines ,Glücksindex" Bescheid, welche Informationen und Indikatoren in welcher Art und Weise in deren Konstruktionen einfließen, ist aber den Wenigsten bekannt. Dementsprechend muss ein besonderes Augenmerk auf die Transparenz der Indexkonstruktion sowie die Kommunikation der beinhalteten Informationen und Methoden gelegt werden. Nur so kann einem späteren Missbrauch des Index als Politikinstrument vorgebeugt werden. Ein Mobilitätsindex muss also neben seiner reinen Berechnung auch immer aufklären, welche Indikatoren seinen Wert beeinflussen. Nur so ist es überhaupt möglich Indizes in Planungsprozessen als vollwertiges Bewertungsverfahren zu integrieren. Das zweite Risiko eines Index besteht darin, dass er durch die Simplizität des quantitativen Werts eine objektive Wahrheit suggeriert, die so tatsächlich gar nicht existiert. Besonders bei der Indizierung von sozialen oder kulturellen Phänomenen, die zu großen Anteilen durch qualitative Kriterien geprägt sind, ist eine Quantifizierung zwangsläufig eine Verkürzung der Realität und wird von einigen Vertretern dieser Disziplinen deshalb strikt abgelehnt (Wilde 2014, S. 33). Problematisch hierbei stellt sich besonders die Suggestion von Objektivität dar, die ubiquitär mit quantitativen Werten assoziiert wird. Deswegen gilt es insbesondere bei der Indexkonstruktion exakt den Qualitätskriterien der Wissenschaft im Allgemeinen und den Ansprüchen der empirischen Sozialforschung im Speziellen zu entsprechen. Anforderungen, denen bisherige Indizes zur Mobilität in keiner Weise gerecht werden. ${ }^{5}$

\subsection{Ansprüche und Qualitätskriterien einer adäquaten Indexkonstruktion}

Für die Indexkonstruktion gelten die grundlegenden wissenschaftlichen Gütekriterien, besonders hervorzuheben sind aber Validität, Reliabilität und Transparenz (Pickel und Pickel 2012, S. 4). Die Validität eines theoretischen oder

\footnotetext{
${ }^{5}$ Hierbei sind insbesondere auf den Bundesländerindex Mobilität der Allianz pro Schiene und den Urban Mobility Readiness Index der Berkley Universität hinzuweisen, welche beide weder den Ansprüchen der Mobilitätsforschung noch der Indexkonstruktion gerecht werden.
} 
empirischen Modells stellt sicher, dass es de facto auch das abbildet, was es vorgibt abzubilden. Beispielsweise sollte ein Index für Mobilität auch tatsächlich alle Teildimensionen von Mobilität abbilden und nicht nur das Verkehrsaufkommen oder die Erreichbarkeit. Für die Indexkonstruktion gilt es hierfür zwei Validitätsebenen zu beachten (Kromrey et al. 2016, S. 186). Erstens die semantische Gültigkeit: Wird eine Begriffsdefinition in adäquate Indikatoren übersetzt? Zweitens die empirische Gültigkeit: Wird auch wirklich ein bestimmter Indikator gemessen?

Die Reliabilität in der empirischen Sozialforschung steht für die Stabilität der Messwerte beziehungsweise dem Ausmaß der Streuung bei wiederholtem Messen (Atteslander 2010, S. 229). Prüft die Validität die adäquate Verknüpfung von Theorie und Empirie, so stellt die Reliabilität die Adäquanz der Messverfahren sicher. Besonders bei einem Index, der eine allgemeingültige und langfristige Auswertungsmethode darstellt, ist es wichtig, dass er immer wieder zuverlässige Aussagen generiert. Die Reliabilität spielt damit besonders bei der Operationalisierung der Indikatoren eine zentrale Rolle und sollte in regelmäßigen Abständen überprüft werden.

Das mit Abstand elementarste Gütekriterium bei der Indexkonstruktion ist die Transparenz. Besonders, da es sich um kein objektiv-statistisches Auswertungsverfahren handelt, ist es von umso größerer Bedeutung den argumentativkonstruktiven Prozess in seiner Vollständigkeit offenzulegen. Jede Indexbildung stellt eine Datenreduktion dar, weshalb die Aussagekraft eines Index immer stark von der verwendeten Methodik abhängt (Rohwer und Pötter 2002, S. 65 ff.). Um einen Index nach wissenschaftlich Gütekriterien bewerten zu können, braucht es deshalb Einblick in die verwendeten Methoden und Daten. Neben der theoretischen Fundierung ist die Transparenz damit eine zentrale Anforderung an die wissenschaftliche Indexbildung (Latcheva und Davidov 2014, S. 752).

Können die Gütekriterien der Validität, Reliabilität und Transparenz in der Regel erst bei der Umsetzung geprüft werden, existieren auch allgemeine Ansprüche aus den erfahrungswissenschaftlichen Theorien, welche den Rahmen der Indexkonstruktion abstecken. Helmut Kromrey et al. (2016, S. 163) formulieren hierfür vier fundamentale Anforderungen, die es auch für einen Mobilitätsindex zu beachten gilt:

1. Die Theorie braucht einen empirischen Bezug und muss überprüfbar sein.

2. Die Begriffe in der Theorie müssen präzise definiert sein.

3. Die Begriffe müssen mit entsprechenden Indikatoren verknüpft sein.

4. Die Begriffe müssen operationalisierbar sein bzw. Beobachtungsoperationen angegeben werden. 
Übertragen auf den Mobilitätsindex bedeutet dies, dass zunächst die identifizierten Einflussdimensionen auf Mobilität mit praktischen Untersuchungen belegt werden müssen. Hierzu muss klar definiert werden, was der Begriff Mobilität genau bedeutet und weitergehend was eine hohe und was eine niedrige Mobilität ausmacht. Erst auf Basis dieses normativen Konstrukts von Mobilität kann im Anschluss eine Indexbildung durchgeführt werden. Zentrale Anforderung für die wissenschaftliche Validität des Index ist anschließend, dass die normative Definition immer transparent mit dem Index zusammen kommuniziert wird. Nur so kann gewährleistet werden, dass der Index seine eigentliche Zielfunktion weiterhin erfüllt. Umgekehrt können Politik und Planung dann entscheiden, inwieweit sie diese normative Definition von Mobilität mittragen und infolgedessen den Index als Methodik nutzen wollen oder nicht. Neben dem Mobilitätsbegriff müssen aber ebenfalls auch die Teildimensionen präzise definiert und mit latenten und messbaren Indikatoren verknüpft werden. Nur dann ist es überhaupt möglich vom komplexen Konstrukt der Mobilität bis hin zu einzelnen Messindikatoren, wie der fußläufigen Entfernung zum Supermarkt oder der Zufriedenheit mit der Wohnumgebung, eine kausal-analytische Zusammenhangsbeziehung zu erstellen (siehe Abb. 3). Diese Zusammenhangsbeziehung gilt es transparent in Form von Indikatoren-Sets, Korrespondenz- und Aggregationsregeln darzustellen. Die Korrespondenzregeln stellen in diesem Sinne die normativen Chiffren dar, welche die Indikatoren-Sets mit dem darzustellenden Phänomen verknüpfen. So könnte beispielsweise die durchschnittliche wöchentliche Arbeitszeit der Bevölkerung in positiver oder negativer Weise mit einem nationalen Produktionsindex korrespondieren, je nachdem welche normativen Vorstellungen sich dahinter verbergen. Auch die Aggregationsregeln stellen durch die Nutzung eines spezifischen statistischen Berechnungsverfahren, beispielsweise der geometrischen Mittelung (UN 2018, S. 33), eine weitere normative Komponente von Indizes dar.

$\mathrm{Zu}$ erwähnen bleibt noch, dass bei der Indexkonstruktion weniger allgemeingültige Konstruktionsregeln eine Rolle spielen als vielmehr das theoretische

\section{Korrespondenzregel $\quad$ 1. Aggregationsregel $\quad 2$ Aggregationsregel}

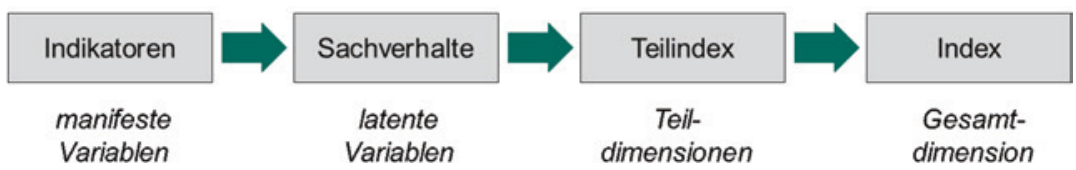

Abb. 3 Kausalitätsprinzip bei der Konstruktion eines mehrdimensionalen Indizes. (Eigene Darstellung) 
Konzept und die Modellierung dieses Konzeptes (OECD 2008, S. 14). Dies bedeutet, dass abhängig vom Untersuchungsgegenstand gänzlich unterschiedliche Aggregations- und Bewertungsregeln genutzt werden können, je nachdem ob Bildung, Glück oder Mobilität indiziert werden sollen. Dementsprechend liegt die Rechtfertigung eines Indizes am Ende auch in der Wahrnehmung und Akzeptanz seines Publikums. Besonders deshalb gilt es, die Gütekriterien der Validität, Reliabilität und Transparenz in besonderem Maße zu beachten und den allgemeinen Ansprüchen an die empirische Sozialforschung jederzeit zu genügen.

\section{Ansprüche an wissenschaftliche Mobilitätsindizes}

Mobilität ist ein komplexes soziales Phänomen, darin besteht Disziplinen übergreifende Einigkeit. Weiterhin sind Indizes eine geeignete Methodik um komplexe, nicht messbare Phänomene darstellbar und vergleichbar zu machen. Es liegt also nahe, auch die Mobilität in Form eines Index darzustellen, insbesondere da es der bisherigen Mobilitätsplanung an konkreten Planungsinstrumenten fehlt. Doch was sind die Ansprüche, um die Mobilität in Form eines Index $\mathrm{zu}$ operationalisieren? Welche Indikatoren können dafür genutzt werden und in welcher Form sind diese zu erheben? Diese und weitere Fragen sollen im Folgenden ausführlich diskutiert werden. Dabei werden die Ansprüche an einen wissenschaftlich validen Mobilitätsindex skizziert, der im Gegensatz zu den bereits existierenden Verkehrs- und Mobilitätsindizes sowohl das gesamte Spektrum der Mobilitätsforschung wie auch die Gütekriterien der Indexbildung abdeckt. Ziel ist es, auch für die Mobilität ein Bewertungsverfahren zu erhalten, das analog zu etablierten Indizes für menschliche Entwicklung oder Demokratie, eine umfassende Analyse und Vergleichbarkeit der Mobilität erlaubt.

\subsection{Mobilitätsspezifische Anforderung an Indikatoren}

Zunächst einmal muss bei einer Operationalisierung der Mobilität für Indizes, aber auch für alle anderen mobilitätsbezogenen Planungsinstrumente, der Vielschichtigkeit und Interdependenz von Mobilität Rechnung getragen werden. Dies bedeutet, dass die Erkenntnisse der unterschiedlichen Disziplinen, was Mobilität darstellt und wie diese beeinflusst wird, mit aufgenommen werden müssen. So müssen jegliche Mess- und Analyseverfahren zur Mobilität strukturelle Rahmenbedingungen, individuelle Faktoren und subjektive Wahrnehmungen erfassen. 
Andernfalls stellen solch reduzierte Verfahren nur unzureichend die komplexe Ausgestaltung der individuellen Möglichkeitsräume dar und sollten nicht für eine adäquate Analyse der Mobilität verwendet werden (siehe Fußnote 5). Die mobilitätsspezifischen Anforderungen für eine Operationalisierung lassen sich auf drei zentrale Punkte herunterbrechen.

\section{Untersuchung der strukturellen Rahmenbedingungen von Mobilität}

Für die Analyse und Beeinflussung individueller Möglichkeitsräume ist die Untersuchung der strukturellen Rahmenbedingungen fundamental. Diese Strukturen legen in physischer und sozioökonomischer Form fest, welche Orte ein Idividuum erreichen kann oder nicht. Hierzu gehört insbesondere die gebaute Umwelt, mit ihren Versorgungs- und Verkehrsstrukturen. Zudem beeinflussen auch ökologische Faktoren, wie Luft- und Lärmbelastung oder eine hohe Verkehrsunsicherheit die individuellen Möglichkeiten. Weiterhin entscheiden ökonomische Faktoren, wie die Kosten für unterschiedliche Verkehrsmittelnutzungen oder die Verfügbarkeit der Arbeitsplätze, darüber, welche Orte ein Individuum aufsuchen kann oder muss. Zur Erfassung dieser strukturellen Rahmenbedingungen existieren bereits unterschiedliche Verfahren, wie die Erreichbarkeitsanalyse (Schwarze 2015), die Umweltanalyse (SenStadtUm 2017) oder Pendelerhebungen (Dauth und Haller 2016). Zusammengenommen bieten diese Verfahren einen umfassenden Einblick in die strukturellen Rahmenbedingungen individueller Mobilität und bilden damit eine zentrale Anforderung mobilitätsspezifischer Untersuchungen.

2. Untersuchung der individuellen Handlungsvoraussetzungen

Wenn es darum geht, die Möglichkeitsräume der Menschen zu beschreiben, spielen zudem die individuellen Voraussetzungen für das eigene Handeln eine zentrale Rolle. Analog zum Verkehrssystem, bei dem eine Schienenverbindung nur von einer Eisenbahn verwendet werden kann und nicht von einem Pkw, so gilt auch für die räumliche Struktur, dass diese nur unter bestimmten individuellen Voraussetzungen, wie einer ausreichenden Gesundheit, der Fähigkeit zum Führen eines Fahrzeugs oder der Verfügbarkeit von finanziellen Mitteln, genutzt werden kann. Die individuellen Handlungsvoraussetzungen sind sozusagen der Schlüssel, der benötigt wird, um sich bestimmte Orte ,erschließen` zu können. Dementsprechend müssen, neben den strukturellen Rahmenbedingungen auch die individuellen Handlungsvoraussetzungen der Menschen vor Ort untersucht werden, um tatsächlich eine Aussage über die Mobilität treffen zu können. Hierzu gehören soziodemografische Faktoren, wie Alter, Einkommen und Haushaltsstruktur, aber auch soziokulturelle Faktoren, wie das Milieu oder das soziale Netzwerk. All diese 
Einflussfaktoren bestimmen, wie wir uns bewegen und welche Möglichkeiten uns zur Verfügung stehen. Eine vollständige Erfassung der Mobilität bedarf also einer ausführlichen Analyse der individuellen Handlungsvoraussetzungen. Klassische Beispiele aus der Praxis sind hierfür die Querschnitterhebungen, wie das sozioökonomische Panel, Mobilität in Deutschland oder SrV (Ahrens et al. 2014; Britzke und Schupp 2017; Nobis und Kuhnimhof 2017).

3. Untersuchung der subjektiven Wahrnehmungen

Die wohl am häufigsten vernachlässigte Anforderung an eine umfassende Mobilitätsanalyse ist schließlich die Untersuchung der subjektiven Wahrnehmungen. Erst ihre Betrachtung ermöglicht einen Einblick in die tatsächliche Ausgestaltung der individuellen Möglichkeitsräume und offenbart häufig unvorhergesehene Barrieren und Hürden, die Menschen auf Grund spezifischer Wahrnehmungen in ihrem Möglichkeitsraum einschränken (siehe den Beitrag von Daubitz in diesem Band). Ganz wichtig hierbei ist die subjektive Erreichbarkeit, welche die strukturellen Rahmenbedingungen und die individuellen Handlungsvoraussetzungen mit der eigenen Selbstwahrnehmung kontextualisieren. Aber auch die Zufriedenheit mit der Wohnumgebung oder die individuelle Lebenslage stellen wichtige Faktoren dar, welche die Möglichkeiten einschränken oder erweitern können. Zuletzt spielt auch die Wahrnehmung der sozialen Umgebung, wie der Familienkontakt und das Gemeinschaftsgefühl in der Lebensumgebung, eine nicht zu vernachlässigende Rolle dahingehend, welche Möglichkeiten für Ortsveränderungen überhaupt wahrgenommen werden können. Es zeigt sich, dass die subjektive Wahrnehmung von Menschen die objektiven Verhältnisse völlig umkehren kann (Wilde 2014, S. 149 ff.), beispielsweise dann, wenn ein neuer Radstreifen auf der Fahrbahn bestimmte Nutzergruppen ausschließt, da Ängste sie von der Nutzung dieser neuen Infrastruktur abhalten. Dementsprechend können wir die tatsächliche Mobilität der Menschen nur untersuchen, wenn neben den strukturellen Rahmenbedingungen und individuellen Handlungsvoraussetzungen auch die subjektiven Wahrnehmungen analysiert werden. Da dies aber nicht durch quantitative Messverfahren oder Studien in seiner Genauigkeit erfasst werden kann, finden sich bis heute nur wenig Beispiele, die erfolgreich die subjektiven Wahrnehmungen in der praktizierten Planung operationalisieren. Ein Beispiel zur systematischen Erfassung solcher Faktoren ist die ,Vermächtnis-Studie " in Deutschland (Allmendinger 2017) oder die Untersuchungen zur sozialen Exklusion in Australien (Currie et al. 2010). Auch wenn dies eine Herausforderung für die klassische quantitativ geprägte Planung darstellt, ist die Erfassung der subjektiven Wahrnehmungen ein zentrales Element, das die Mobilitätsplanung von der Verkehrs- oder Raumplanung unterscheidet. 
Zusammenfassend können wir festhalten, dass für jedwede Operationalisierung der Mobilität drei zentrale Dimensionen betrachtet werden müssen: die Strukturen, die Individuen und ihre Wahrnehmungen. Nur wenn alle drei Dimensionen in Erhebung, Analyse und Auswertung einbezogen wurden, kann von einer vollwertigen Mobilitätsplanung gesprochen werden. Insofern gilt auch für Verfahren, welche die Mobilität messbar oder bewertbar machen, dass alle drei Dimensionen untersucht werden müssen. Ein Mobilitätsindex benötigt also Indikatoren zu den strukturellen Rahmenbedingungen, den individuellen Handlungsvoraussetzungen sowie zu den subjektiven Wahrnehmungen.

\subsection{Indexspezifische Anforderung an Indikatoren}

Wie bereits im Abschnitt zur Indexmethodik angedeutet, bringt auch die Konstruktion eines Indizes spezifische Anforderungen mit sich. So ist die Indexkonstruktion ein Auswertungsverfahren, das bereits gemessene Indikatoren regelbasiert zusammenführt. Die Funktionsweise eines Index liegt insbesondere in der großräumlichen Anwendbarkeit und entfaltet seine Wirkung erst, wenn ausreichend Vergleichsräume einbezogen werden können. Weiterhin enthält jeder Index auch eine normative Komponente dahin gehend, dass durch seine Korrespondenz- und Aggregationsregeln festgelegt wird, welche Indikatoren eine positive oder eine negative Wirkung auf den Indexwert entfalten. Insofern nimmt ein Index immer auch eine normative Klassifizierung aller enthaltenen Indikatoren vor. Auf Basis der sozialwissenschaftlichen Gütekriterien für die Indexmethodik lassen sich folgende vier Anforderungen für die Indikatoren identifizieren:

\section{Relevanz}

Indikatoren, die in irgendeiner Form zur Erklärung von Syndromen oder Phänomenen herangezogen werden, müssen einen relevanten Einfluss auf die zu erklärende Größe haben. Dies bedeutet, dass für die jeweiligen Indikatoren aufgezeigt werden muss, dass sie eigenständig auf die zu erklärende Größe einwirken und dabei keine Multikollinearität besteht. Nur wenn ein Indikator unabhängig von anderen Indikatoren einen positiven oder negativen Einfluss auf die Indexgröße entfaltet, ist dieser als relevant einzustufen. Bevor ein Indikator also für einen Index verwendet werden kann, muss zuvor in Praxisuntersuchungen, beispielsweise mit Hilfe von Regressionsanalysen, nachgewiesen werden, dass dieser relevant und ohne signifikante Korrelation auf die zu erklärende Größe einwirkt. 
2. Messbarkeit

Ein weiterer Anspruch an Indikatoren, die innerhalb eines Index verwendet werden sollen, ist die Messbarkeit und die daran anschließende Quantifizierbarkeit. Insbesondere da Indizes in der Regel komplexe, nicht naturwissenschaftlich messbare Phänomene beschreiben, ist es wichtig, dass die konstituierenden Größen eindeutig quantifizierbar sind und damit das nicht messbare Phänomen auch tatsächlich indizieren können. Dies bedeutet, dass qualitative Methoden und Indikatoren nur für die Indexberechnung verwendet werden können, wenn die Informationen in einem Schritt reliabel zu quantifizieren sind. Nur wenn die Indikatoren nach dem Anspruch der Reliabilität wiederholt messbar sind, können sie für einen Index verwendet werden.

3. Aggregierbarkeit

Der Anspruch der Aggregierbarkeit ist ein typisches Charakteristikum von Indexindikatoren, da die Indexmethodik maßgeblich in der Zusammenführung von Daten und Informationen besteht. Dies bedeutet, dass die Indikatoren großflächig erfassbar sein müssen, um ausreichend Informationen für eine Aggregation zur Verfügung zu stellen. Auch dieses Kriterium erschwert es, aufwendig zu erhebende qualitative Indikatoren für einen Index zu nutzen, da ihre Aggregierbarkeit nur durch übermäßigen Erhebungsaufwand zu realisieren ist. Ein Indikator gilt also nur als aggregierbar, wenn er in großer Anzahl (teil-)repräsentativ erhoben werden kann.

4. Bewertbarkeit

Die vierte und zentralste Anforderung an Indikatoren bei der Indexkonstruktion ist ihre Bewertbarkeit. Hierin kommt die Normativität des Verfahrens zur Geltung, in dem transparent den einzelnen Indikatoren eine positive oder negative Wirkung auf die zu erklärende Größe zugeteilt wird. Diese Zuteilung muss selbstverständlich transparent und nachvollziehbar, aber auch evidenzbasiert erfolgen. Dies bedeutet, dass in Praxisuntersuchungen nachgewiesen werden muss, weshalb der jeweilige Indikator neben seiner Relevanz auch ordinal oder metrisch bewertet werden kann. Die ordinale Bewertbarkeit von Indikatoren äußert sich dahin gehend, dass bei mindestens zwei Messwerten eindeutig bestimmt werden kann, welcher ,besser" und welcher ,schlechter' in Bezug auf die Indexgröße ist. Erst durch diese normative Chiffrierung der Indikatoren ist eine Nutzung im Rahmen von Indizes möglich. Bevor ein Indikator also für einen Index verwendet werden kann, muss seine Bewertbarkeit transparent aufgezeigt und nachvollziehbar dargelegt werden. 
Diese vier indexspezifischen Anforderungen an die Operationalisierung mit Indikatoren führen dazu, dass mitunter eine große Zahl von Einflussgrößen auf Syndrome oder Phänomene nicht in das Indikatoren-Set mit aufgenommen werden kann. Dies begründet sich damit, dass Indizes immer nur Modellkonstruktionen sind, sie also nicht präzise messbare Phänomene beschreiben, wie menschliche Entwicklung, Demokratie oder eben Mobilität. Diese Simplifizierung geht mit einer rigorosen Indikatorenreduktion einher - insbesondere für qualitative Faktoren -, die dazu führt, dass am Ende nur wenige Indikationsgrößen, welche den vier dargestellten Anforderungen entsprechen, für einen möglichen Index verwendet werden können.

\section{Fazit}

Ausgehend von der Frage, inwiefern uns die Indexmethodik dabei unterstützen kann, eine systematische Messung von Mobilität besser in der deutschen Planungspraxis zu integrieren und damit das Leitbild einer öffentlichen Mobilität auch methodisch mit Leben zu füllen, wurden die Anforderungen von Mobilität und von Indizes hinsichtlich einer Operationalisierung diskutiert. Dabei wurde gezeigt, dass sowohl die Mobilität als auch die Indexmethodik klare Ansprüche an die auszuwählenden Indikatoren stellt. Diese Ansprüche gilt es zukünftig für einen möglichen Mobilitätsindex zusammenzuführen, um den bereits kursierenden Mobilitätsindizierungsverfahren eine wissenschaftlich valide Methode entgegenzusetzen. Hierzu muss die Interdisziplinarität der Mobilität mit den Qualitätskriterien der Indexkonstruktion zusammengeführt werden. Solch einen komplexen Index an dieser Stelle bereits vorzulegen, würde den Rahmen dieses Artikels sprengen. Vielmehr spielen zunächst nur die wissenschaftlichen Ansprüche einer indexbasierten Mobilitätsmessung im Allgemeinen eine Rolle. Die konkreten Ausgestaltungsmöglichkeiten eines Mobilitätsindex bleiben abhängig von Erkenntnisinteresse, Vergleichsraum und Datenverfügbarkeit - an dieser Stelle offen. So könnte ein Mobilitätsindex auf der einen Seite genutzt werden, um großflächige Vergleiche zwischen den Gemeinden und Städten in Europa zu ermöglichen, analog zum Human Development Index. Ziel eines solchen Index wäre die Mobilität zu einer politischen Zielgröße zu erheben und somit eine starke normative Optimierungsdynamik zu forcieren. Auf der anderen Seite könnte ein möglicher Mobilitätsindex kleinräumlich auf kommunaler Ebene genutzt werden, um mögliche ,Brennpunkte' in der eigenen Kommune zu identifizieren. In dieser Form könnte der Index stärker partizipativ konzipiert werden, indem beispielsweise die Bürgerinnen und Bürger vor Ort über die relevanten Indikatoren entscheiden. 
Für alle Operationalisierungsmöglichkeiten gilt, dass alle drei konstituierenden Ebenen der Mobilität in der Untersuchung einbezogen werden. Dazu zählen die strukturellen Rahmenbedingungen in Raum und Gesellschaft, die individuellen Handlungsvoraussetzungen der Menschen sowie die subjektiven Wahrnehmungen der eigenen Möglichkeitsräume. Erst wenn Indikatoren aus allen drei Dimensionen in der Analyse einbezogen werden, lässt sich von einer vollwertigen Mobilitätsmessung sprechen. Dies gilt in gleicher Form für jegliche praktizierte Mobilitätsplanung: Verfahren und Methoden, die dabei unterstützen sollen, eine öffentliche Mobilität adäquat zu etablieren, müssen also räumliche und soziale Strukturen, demografische und soziokulturelle Handlungsvoraussetzungen sowie subjektive Wahrnehmungen einbeziehen.

Die vorgestellte Methodik der Indexkonstruktion stellt einen Ansatz dar, wie diese Mobilitätsindikatoren innerhalb einer Bewertungsmethodik zusammengeführt werden können. Ein wissenschaftlich valides Indexverfahren bietet uns die Möglichkeit, Ansprüche und Leitbilder, wie die einer öffentlichen Mobilität, transparent nachzuverfolgen und Handlungsbedarfe oder Entwicklungspotenziale aufzuzeigen. Hierfür gilt es jedoch, die Qualitätsansprüche bezüglich des zugrunde liegenden Indikatoren-Sets zu beachten, damit ein Mobilitätsindex den Ansprüchen wissenschaftlicher Verfahren nach Transparenz, Reliabilität und Validität entspricht. Die Indikatoren für einen Mobilitätsindex müssen demnach alle relevant, messbar, aggregierbar und bewertbar sein. Nur dann kann von einem wissenschaftlichen Mobilitätsindex gesprochen werden. Führen wir diese indexspezifischen Ansprüche mit denen der Mobilitätsforschung zusammen, erhalten wir eine Checkliste für das Indikatoren-Set zur Mobilitätsmessung (siehe Abb. 4). Diese Checkliste gibt Mobilitätsplanenden einen Hinweis darauf, wie sie einen eigenen Mobilitätsindex konstruieren können und welche Indikatoren hierfür zu untersuchen sind.

\begin{tabular}{|l||c|c|c|c|}
\hline \multicolumn{7}{|c|}{ Auswahlkriterien Indikatoren-Set Mobilitätsmessung } \\
\hline Indikatoren für die... & relevant & messbar & aggregierbar & bewertbar \\
\hline strukturellen Rahmenbedingungen & $\square$ & $\square$ & $\square$ & $\square$ \\
\hline individuellen Handlungsvoraussetzungen & $\square$ & $\square$ & $\square$ & $\square$ \\
\hline subjektiven Wahrnehmungen & $\square$ & $\square$ & $\square$ & $\square$ \\
\hline
\end{tabular}

Abb. 4 Beispielhafte Checkliste für die Erfassung valider Indikatoren zur Mobilitätsmessung. (Eigene Darstellung) 
Abschließend zeigen diese Betrachtungen zu einem Mobilitätsindex anschaulich, mit welchen Herausforderungen die Messung der Mobilität innerhalb standardisierter Planungs- und Bewertungsverfahren konfrontiert ist. Insbesondere die Vielschichtigkeit von Mobilität bringt eigene Anforderungen mit sich, die mobilitätsbezogene Verfahren berücksichtigen müssen. Damit unterscheidet sich die Mobilitätsplanung grundlegend von bereits etablierten Verfahren aus der Raum- oder Verkehrsplanung. Mobilität bringt strukturelle Faktoren zusammen mit individuellen Voraussetzungen und subjektiven Wahrnehmungen. Damit erhöht sich auf der einen Seite zwar die Komplexität des Planungsfelds, dies ermöglicht aber auf der anderen Seite neue Handlungsspielräume, um die Mobilität zielorientiert zu beeinflussen und eine Öffentliche Mobilität zu operationalisieren. Indizes bieten sich hierbei als passende Verfahren an, Licht in die Black Box der individuellen Möglichkeitsräume zu bringen und die Mobilität damit evidenzbasiert gestaltbar zu machen. Fest steht, dass sowohl die Mobilität als auch mögliche Verfahren ihre spezifischen Anforderungen an eine Operationalisierung mitbringen. Diese Anforderungen müssen akribisch bei der Konzeption neuer Planungsverfahren berücksichtigt werden. Ansonsten steht die Mobilität weiterhin nur als leere Worthülse vor reduktionistischen Verfahren der Verkehrs- und Raumplanung, in denen weder individuelle Belange noch subjektive Wahrnehmungen eine Rolle spielen.

\section{Literatur}

Ahrens, Gerd-Axel, F. Ließke, R. Wittwer, S. Hubrich und S. Wittig. 2014. Methodenbericht zum Forschungsprojekt „Mobilität in Städten-SrV 2013“. In: Im Auftrag von Städten, Verkehrsunternehmen, Verkehrsverbünden und Bundesländern, bearbeitet durch die Technische Universität Dresden, Lehrstuhl Verkehrs-und Infrastrukturplanung.

Allmendinger, Jutta. 2017. Das Land, in dem wir leben wollen. Wie die Deutschen sich ihre Zukunft vorstellen. München: Pantheon.

Altenburg, Sven, P. Gaffron, C. Gertz. 2009. Teilhabe zu ermöglichen bedeutet Mobilität zu ermöglichen. Diskussionspapier des Arbeitskreises Innovative Verkehrspolitik der Friedrich-Ebert-Stiftung.

Atteslander, Peter. 2010. Methoden der empirischen Sozialforschung. Berlin: Erich Schmidt Verlag.

BBSR. 2020. Erreichbarkeitsmodell des BBSR. Bundesinstitut für Bau-, Stadt und Raumforschung: https://www.bbsr.bund.de/BBSR/DE/Raumbeobachtung/UeberRaumbeobachtung/Komponenten/Erreichbarkeitsmodell/erreichbarkeitsmodell_node.htm. Zugegriffen 20.07.2020.

Becker, Udo J. 2018. Verkehr und Umwelt. In: Schwedes, Oliver (Hg.): Verkehrspolitik. Wiesbaden: Springer VS, S. 71-88. 
Beckmann, Klaus J., M. Hesse, C. Holz-Rau, M. Hunecke. 2006. StadtLeben - Wohnen, Mobilität und Lebensstil. Neue Perspektiven für Raumund Verkehrsentwicklung. Wiesbaden: VS Verlag für Sozialwissenschaften/GWV Fachverlage GmbH Wiesbaden.

BMBF. 2019. Karliczek: Wir gestalten Mobilität nachhaltig - mit Städten und Gemeinden gehen wir in der Forschung voran. Im Rahmen des Wettbewerbs „,MobilitätsWerkStadt 2025 " nehmen 50 Mobilitätsmanager Arbeit auf. Berlin.

Britzke, Janina, J. Schupp. 2017. SOEP Wave Report 2017. https://www.diw.de/documents/ publikationen/73/diw_01.c.592826.de/wave_report_2017.pdf Zugegriffen 20.07.2020.

Busch-Geertsema, Annika, M. Lanzendorf, H. Müggenburg, M. Wilde. 2016. Mobilitätsforschung aus der nachfrageorientierten Perspektive: Theorien, Erkenntnisse und Dynamiken des Verkehrshandelns. In: Schwedes, Oliver, W. Canzler, A. Knie (Hg.): Handbuch Verkehrspolitik. Wiesbaden: Springer VS, S. 755-774.

Currie, Grahama, T. Richardson, P. Smyth, D. Vella-Brodrick, J. Hine, K. Lucas, J. Stanley, J. Morris, R. Kinnear, J. Stanley. 2010. Investigating links between transport disadvantage, social exclusion and well-being in Melbourne - Updated results. In: Research in Transportation Economics 29(1), S. 287-295.

Dangschat, Jens S. 2018. Soziale Milieus in der Mobilitätsforschung. In: Barth, Bertram, B. Flaig, N. Schäuble, M. Tautscher (Hg.): Praxis der Sinus-Milieus®. Gegenwart und Zukunft eines modernen Gesellschafts- und Zielgruppenmodells. Wiesbaden: Springer VS, S. 139-153.

Daubitz, Stephan. 2017. Mobilität und soziale Exklusion: Ein Plädoyer für ein zielgruppenspezifisches Mobilitätsmanagement. In: Wilde, Mathias, M. Gather, C. Neiberger, J. Scheiner (Hg.): Verkehr und Mobilität zwischen Alltagspraxis und Planungstheorie. Wiesbaden: Springer Fachmedien Wiesbaden, S. 53-64.

Dauth, Wolfgang, P. Haller. 2016. The valuation of changes in commuting distances. An analysis using georeferenced data. IAB-Discussion Paper. https://hdl.handle. net/10419/148861. Zugegriffen 20.07.2020.

Delbosc, Alexa, G. Currie. 2011. Transport problems that matter - social and psychological links to transport disadvantage. In: Journal of Transport Geography 19(1), S. 170-178.

DeVos, Jonas de, T. Schwanen, V. van Acker, F. Witlox. 2013. Travel and Subjective Well-Being: A Focus on Findings, Methods and Future Research Needs. In: Transport Reviews 33(4), S. 421-442.

FES (Hg.). 2019. Hürden auf dem Weg zur Zukunftsstadt. Strategien für eine integrierte Stadt- und Verkehrspolitik. Berlin: Friedrich Ebert Stiftung.

FGSV - Forschungsgesellschaft für Straßen- und Verkehrswesen. 2015. Hinweise zu Mobilität und sozialer Exklusion. Forschungsstand zum Zusammenhang von Mobilitätsund Teilhabechancen. Köln: FGSV Verlag GmbH.

FGSV - Forschungsgesellschaft für Straßen- und Verkehrswesen. 2020. Organigramm der FGSV. https://www.fgsv.de/organigramm.html. Zugegriffen 20.07.2020.

Götz, Konrad, J. Deffner, T. Klinger. 2016. Mobilitätsstile und Mobilitätskulturen Erklärungspotentiale, Rezeption und Kritik. In: Schwedes, Oliver, W. Canzler, A. Knie (Hg.): Handbuch Verkehrspolitik. Wiesbaden: Springer VS, S. 781-804.

Hine, Julian, M. Grieco. 2003. Scatters and clusters in time and space. Implications for delivering integrated and inclusive transport. In: Transport Policy (4), S. 299-306.

Holz-Rau, Christian. 2019. $\mathrm{CO}_{2}$-Bepreisung und Entfernungspauschale. In: Internationales Verkehrswesen (4), S. 10-12. 
Jarass, Julia. 2012. Wohnstandortpräferenzen und Mobilitätsverhalten. Wiesbaden: VS Verlag für Sozialwissenschaften.

Kiba-Janiak, Maja, J. Witkowski. 2019. Sustainable Urban Mobility Plans: How Do They Work? In: Sustainability 11(17), S. 4605.

Knoflacher, Hermann. 2009. Stehzeuge-Fahrzeuge: der Stau ist kein Verkehrsproblem. Böhlau Verlag Wien.

Kromrey, Helmut; J. Roose, J. Strübing. 2016. Empirische Sozialforschung. Modelle und Methoden der standardisierten Datenerhebung und Datenauswertung mit Annotationen aus qualitativ-interpretativer Perspektive. Konstanz/München: UVK Verlagsgesellschaft mbh; UVK/Lucius.

Kutter, Eckhard. 2010. Mobilitätsforschung - wenig Hilfreich für die Problemlösung im Stadtverkehr. In: Verkehr und Technik, S. 3-7.

Latcheva, Rossalina, E. Davidov. 2014. Skalen und Indizes. In: Baur, Nina, J. Blasius (Hg.): Handbuch Methoden der empirischen Sozialforschung. Wiesbaden: Springer Fachmedien Wiesbaden, S. 745-756.

Lättman, Katrin, M. Friman, L. Olsson. 2016. Perceived Accessibility of Public Transport as a Potential Indicator of Social Inclusion. In: Social Inclusion 4(3), S. 36.

Mäcke, Paul Artur. 1964. Das Prognoseverfahren in der Straßenverkehrsplanung. Gekürzte Fassung d. Habil.-Schr. Aachen, RWTH u.d.T.: Mäcke, Paul Arthur: Algorithmus der Verkehrsprognose. Wiesbaden/Berlin: Bauverl.

Nobis, Claudia, T. Kuhnimhof. 2017. Mobilität in Deutschland - MiD Ergebnisbericht. Studie von infas, DLR, IVT und infas 360 im Auftrag des Bundesministerium für Verkehr und digitale Infrastruktur. Bonn/Berlin.

Nordbakke, Susanne, T. Schwanen. 2015. Transport, unmet activity needs and wellbeing in later life: exploring the links. In: Transportation 42(6), S. 1129-1151.

OECD. 2008. Handbook on Constructing Composite Indicators: Methodology and User Guide. OECD Publishing.

Pickel, Susanne, G. Pickel. 2012. Die Messung von Indizes in der Vergleichenden Politikwissenschaft - methodologische Spitzfindigkeit oder substantielle Notwendigkeit. In: Zeitschrift für Vergleichende Politikwissenschaft 6(S1), S. 1-17.

Rohwer, Götz, U. Pötter. 2002. Methoden sozialwissenschaftlicher Datenkonstruktion. Weinheim: Juventa-Verl.

Rupprecht Consult. 2020. Decision makers summary for Developing and Implementing a Sustainable Urban Mobility Plan. Trialog Publishers.

Scheepers, C. E., G.C.W. Wendel-Vos, E.E.M.M. van Kempen, E. L. de Hollander, H. J. van Wijnen, J. Maas, F.R.J. den Hertog, B.A.M. Staatsen, H. L. Stipdonk, L.L.R. Int Panis, P.J.V. van Wesemael, A. J. Schuit. 2016. Perceived accessibility is an important factor in transport choice - Results from the AVENUE project. In: Journal of Transport \& Health 3(1), S. 96-106.

Scheiner, Joachim. 2016. Verkehrsgenese: Wie entsteht Verkehr? In: Schwedes, Oliver, W. Canzler, Weert, A. Knie (Hg.): Handbuch Verkehrspolitik. Wiesbaden: Springer VS, S. 679-700.

Schlag, Bernhard. 2016. Automatisiertes Fahren im Straßenverkehr - Offene Fragen aus Sicht der Psychologie. In: Zeitschrift für Verkehrssicherheit 62, S. 94-98.

Schwarze, Björn. 2015. Eine Methode zum Messen von Naherreichbarkeit in Kommunen. TU Dortmund: Dissertation. 
Schwedes, Oliver. 2018. Verkehrspolitik als Gesellschaftspolitik. In: ders. (Hg.): Verkehrspolitik. Wiesbaden: Springer VS, S. 3-24.

Schwedes, Oliver, A. Rammert. 2020a. Was ist Integrierte Verkehrsplanung? Hintergründe und Perspektiven einer am Menschen orientierten Planung. IVP-Discussion Paper. 2/2020. Berlin.

Schwedes, Oliver, A. Rammert. 2020b. Mobilitätsmanagement. Ein neues Handlungsfeld integrierter Verkehrspolitik und -planung. Wiesbaden: Springer Gabler.

Schwedes, Oliver, S. Daubitz, A. Rammert, B. Sternkopf, M. Hoor. 2018. Kleiner Begriffskanon der Mobilitätsforschung. Berlin. TU Berlin: Discussion Paper.

Schwedes, Oliver, A. Rammert, B. Sternkopf,. 2017. Mobilitätsmanagement. Möglichkeiten und Grenzen verkehrspolitischer Gestaltung am Beispiel Mobilitätsmanagement. Berlin. TU Berlin: https://www.ivp.tu-berlin.de/fileadmin/fg93/Forschung/Projekte/ Mobilit\%C3\%A4tsmanagement/Endbericht_MobMan.pdf, Abschlussbericht. Zugegriffen 20.07.2020.

SenStadtUm 2017. Basisbericht Umweltgerechtigkeit. Grundlagen für die sozialräumliche Umweltpolitik. Senatsverwaltung für Stadtentwicklung und Umwelt: https://www.berlin. de/senuvk/umwelt/umweltgerechtigkeit/download/umweltgerechtigkeit_broschuere.pdf Zugegriffen 20.07.2020.

Spinney, Jamie E.L., D. Scott, K.B. Newbold. 2009. Transport mobility benefits and quality of life: A time-use perspective of elderly Canadians. In: Transport Policy 16(1), S. $1-11$.

Stanley, John K., D.A. Hensher, J.R. Stanley, D. Vella-Brodrick. 2011. Mobility, social exclusion and well-being: Exploring the links. In: Transportation Research Part A: Policy and Practice 45(8), S. 789-801.

Steierwald, Gerd, H. D. Künne, W. Vogt. 2005. Stadtverkehrsplanung. Berlin, Heidelberg: Springer.

UN. 2018. Human Development Indices and Indicators 2018. United Nations.

Vallée, Dirk (Hg.). 2008. Mobilität und Verkehr managen - aus der Forschung für die Praxis. Tagungsband zum 9. Aachener Kolloquium „Mobilität und Stadt“, AMUS 2008. Aachen: Inst. für Stadtbauwesen und Stadtverkehr.

Voges, Wolfgang, O. Jürgens, A. Mauer, E. Meyer. 2003. Methoden und Grundlagen des Lebenslagenansatzes. Endbericht. Bremen.

Wilde, Mathias. 2014. Mobilität und Alltag. Wiesbaden: Springer Fachmedien Wiesbaden.

Wilde, Mathias. M. Gather, C. Neiberger, J. Scheiner (Hg.). 2017. Verkehr und Mobilität zwischen Alltagspraxis und Planungstheorie. Wiesbaden: Springer Fachmedien Wiesbaden.

Wilde, Mathias, T. Klinger. 2017. Integrierte Mobilitäts- und Verkehrsforschung: zwischen Lebenspraxis und Planungspraxis. In: Wilde, Mathias, M. Gather, C. Neiberger, J. Scheiner, Joachim (Hg.): Verkehr und Mobilität zwischen Alltagspraxis und Planungstheorie. Wiesbaden: Springer Fachmedien Wiesbaden, S. 5-23.

Wulfhorst, Gebhard, M. Wolfram. 2010. Lernen von Frankreich? Die „Plans de Déplacements Urbains“ als Beispiel inkrementeller Steuerung. In: IÖR Texte (Steuerung einer nachhaltigen Verkehrsentwicklungsplanung in Deutschland), S. 23-26. 
Open Access Dieses Kapitel wird unter der Creative Commons Namensnennung 4.0 International Lizenz (http://creativecommons.org/licenses/by/4.0/deed.de) veröffentlicht, welche die Nutzung, Vervielfältigung, Bearbeitung, Verbreitung und Wiedergabe in jeglichem Medium und Format erlaubt, sofern Sie den/die ursprünglichen Autor(en) und die Quelle ordnungsgemäß nennen, einen Link zur Creative Commons Lizenz beifügen und angeben, ob Änderungen vorgenommen wurden.

Die in diesem Kapitel enthaltenen Bilder und sonstiges Drittmaterial unterliegen ebenfalls der genannten Creative Commons Lizenz, sofern sich aus der Abbildungslegende nichts anderes ergibt. Sofern das betreffende Material nicht unter der genannten Creative Commons Lizenz steht und die betreffende Handlung nicht nach gesetzlichen Vorschriften erlaubt ist, ist für die oben aufgeführten Weiterverwendungen des Materials die Einwilligung des jeweiligen Rechteinhabers einzuholen.

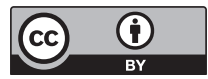

